\title{
Impact of intercensal population projections and error of closure on breast cancer surveillance: examples from 10 California counties
}

\author{
Amanda I Phipps ${ }^{1,2}$, Christina A Clarke ${ }^{3}$ and Rochelle R Ereman ${ }^{1}$
}

\author{
${ }^{1}$ Marin County Department of Health and Human Services, Epidemiology Program, San Rafael, California, USA \\ ${ }^{2}$ Currently working at the Northern California Cancer Center, Fremont, California, USA \\ ${ }^{3}$ Northern California Cancer Center, Fremont, California, USA \\ Corresponding author: Rochelle R Ereman, rereman@co.marin.ca.us
}

Received: 9 Sep 2004 Revisions requested: 23 Nov 2004 Revisions received: 25 Apr 2005 Accepted: 11 May 2005 Published: 7 Jun 2005

Breast Cancer Research 2005, 7:R655-R660 (DOI 10.1186/bcr1266)

This article is online at: http://breast-cancer-research.com/content/7/5/R655

(C) 2005 Phipps et al.; licensee BioMed Central Ltd.

This is an Open Access article distributed under the terms of the Creative Commons Attribution License (http://creativecommons.org/licenses/by/ 2.0), which permits unrestricted use, distribution, and reproduction in any medium, provided the original work is properly cited.

\begin{abstract}
Introduction In 2001, data from the California Cancer Registry suggested that breast cancer incidence rates among nonHispanic white (nHW) women in Marin County, California, had increased almost 60\% between 1991 and 1999. This analysis examines the extent to which these and other breast cancer incidence trends could have been impacted by bias in intercensal population projections.
\end{abstract}

\begin{abstract}
Method We obtained population projections for the year 2000 projected from the 1990 census from the California Department of Finance (DOF) and population counts from the 2000 US Census for $\mathrm{nHW}$ women living in 10 California counties and quantified age-specific differences in counts. We also computed age-adjusted incidence rates of invasive breast cancer in order to examine and quantify the impact of differences between the population data sources.
\end{abstract}

Results Differences between year 2000 DOF projections and year 2000 census counts varied by county and age and ranged from underestimates of $60 \%$ to overestimates of $64 \%$. For Marin County, the DOF underestimated the number of $\mathrm{nHW}$ women aged 45 to 64 years by $32 \%$ compared to the 2000 US census.
This difference produced a significant 22\% discrepancy between breast cancer incidence rates calculated using the two population data sources. In Los Angeles and Santa Clara counties, DOF-based incidence rates were significantly lower than rates based on census data. Rates did not differ significantly by population data source in the remaining seven counties examined.

Conclusion Although year 2000 population estimates from the DOF did not differ markedly from census counts at the state or county levels, greater discrepancies were observed for racestratified, age-specific groups within counties. Because breast cancer incidence rates must be calculated with age-specific data, differences between population data sources at the agerace level may lead to mis-estimation of breast cancer incidence rates in county populations affected by these differences, as was observed in Marin County. Although intercensal rates based on population projections are important for timely breast cancer surveillance, these rates are prone to bias due to the error of closure between population projections and decennial census population counts. Intercensal rates should be interpreted with this potential bias in mind.

\section{Introduction}

From the inception of the Surveillance, Epidemiology, and End Results (SEER) national cancer registry network in 1973, Marin County, California, a small county near San Francisco, has consistently reported higher than average annual incidence rates of breast cancer. Averaged from 1973 to 1999 , Marin County reported the highest overall breast cancer incidence rate of the 199 counties included in the SEER database (based on the SEER 9 November 2001 submission released April 2004) [1]. In recent years, reports of rapidly increasing breast cancer rates in Marin County attracted public and media attention. These reports suggested that overall ageadjusted incidence rates of invasive breast cancer in non-Hispanic white (nHW) women living in Marin County had increased approximately 60\% between 1990 and 1999, as compared to $5 \%$ in surrounding regions (Fig. 1) [2]. These 
Figure 1

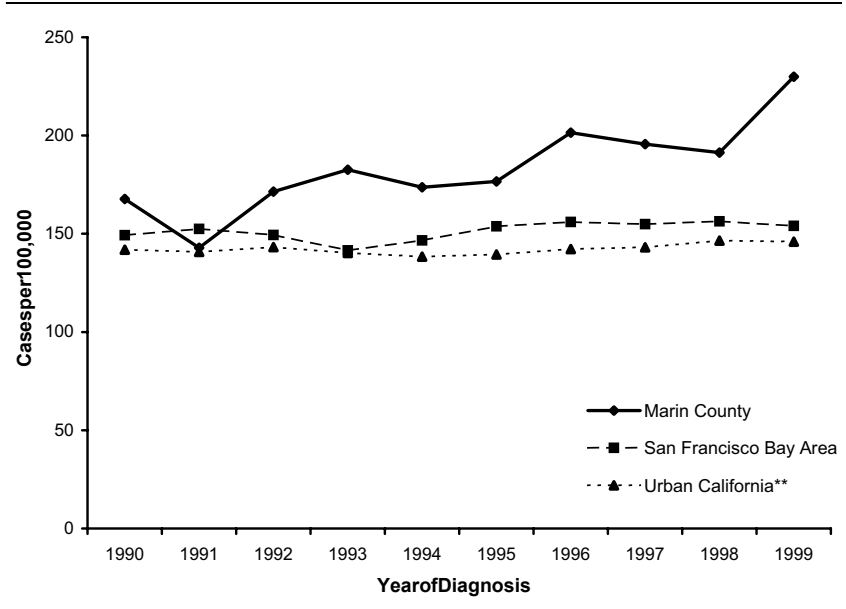

Breast cancer incidence in Marin County, California, and surrounding areas based on Department of Finance population projections for 1990 to 1999 . Rates are age-adjusted to the 2000 US standard population, based on population data unadjusted to the 2000 US census. ${ }^{* \star}$ Case data from the California Cancer Registry.

trends have resulted in Marin County having one of the highest incidence rates reported in the world and have prompted public and scientific concern.

Several possible explanations have been suggested for these breast cancer incidence patterns. Overall, the socio-demographic profile of most women living in Marin County would suggest a higher prevalence of women with known risk factors for breast cancer: relatively high proportions of the county population are of $\mathrm{nHW}$ ethnicity and college-educated, and the county has a median household income almost double the national average (derived from data from Population estimates, 2000 Census of Population and Housing, 1990 Census of Population and Housing, Small Area Income and Poverty Estimates, County Business Patterns, 1997 Economic Census, Minority- and Women-Owned Business, Building Permits, Consolidated Federal Funds Report, 1997 Census of Governments [3]). In addition, women living in Marin County have fewer children, report a later age at first childbirth, and have higher rates of alcohol consumption than most areas of California, all of which correspond to an increased risk of developing breast cancer [4-6].

Another possible explanation for the observed increase in breast cancer incidence in Marin County during the 1990s involves error in population estimates used in the calculation of cancer rates. Intercensal population estimates, as are used to calculate breast cancer incidence rates for Marin County, are used by a variety of health surveillance organizations nationwide. In order to track changes in the occurrence of health outcomes in a timely manner, disease registries, vital statistics agencies, and local health departments must rely on timely estimates of annual population size; however, for most locales in the United States, the population is counted only once every 10 years as part of the national census. Population estimates for intercensal years are projected from census counts from the most recent decennial census along with other governmental data (e.g., vital statistics and immigration records), and are subject to adjustment after the release of data from the subsequent census. The discrepancy between year 2000 population data from the 2000 census and population projections for the year 2000 based on the 1990 census is known as the 'error of closure'.

In California, intercensal population projections are available from two sources, the US Census Bureau and the California Department of Finance (DOF). Although it is uncertain which agency produces more accurate population projections, most California health agencies rely on data from the DOF, perhaps because the methodology used by the census includes little county-specific information, because significant flaws in Census Bureau-produced estimates have been cited in the past, and because the DOF incorporates additional county- and state-specific information into population projections [7-9]. In order to project the size of the population of California by county, gender, race/ethnicity, and 1 year age increments, for the next 50 years, the DOF not only uses data from the most recent national census, but also enhanced state data resources, such as state records of drivers license change of address transactions, migration patterns based on previous censuses, ethnic group-specific fertility rates, information from the Department of Corrections regarding the capacity and flow of prisoners through facilities, and information from the Pentagon to predict military base closures and reassignments. The DOF also makes adjustments to all intercensal population projections dating back to the previous national census with the release of new national census counts $[9,10]$.

Despite the detailed algorithm used by the DOF to project the distribution and size of the California population, these projections are subject to the same limitations as intercensal population figures generated by the Census Bureau; the risk of inaccuracy increases as annual estimates become more temporally removed from the most recent census. Furthermore, estimates for small areas, or certain age, gender, and racial/ ethnic groups are prone to even larger biases due to algorithm inaccuracies: areas with a high growth rate, a large population of retirees, or a large population of foreign-born individuals are likely to be underestimated, while areas with high poverty, and areas with a negative growth rate are likely to be overestimated [7].

The following analysis was conducted to assess the impact on breast cancer incidence rates of the error of closure in stratified DOF projections, 10 years removed from the most recent national census. The goals of this analysis were: to examine how closely population estimates for the year 2000 from the 
Table 1

Percent difference between DOF and census population estimates for non-Hispanic white women by age (2000)

\begin{tabular}{|c|c|c|c|c|c|c|c|c|c|c|}
\hline Age (years) & Alameda & Contra Costa & Marin & San Francisco & San Mateo & Monterey & San Benito & Santa Clara & Santa Cruz & Los Angeles \\
\hline All ages & 3.4 & 3.8 & -1.4 & -5.7 & 3.8 & 4.0 & 10.4 & 8.7 & 4.7 & 2.1 \\
\hline Under 5 & 8.5 & -1.1 & -5.1 & 23.2 & 1.5 & 19.2 & 6.0 & 5.2 & 11.5 & 0.8 \\
\hline $5-14$ & 13.2 & $<0.1$ & -1.5 & 64.1 & 9.2 & 27.7 & -6.7 & 10.4 & 10.8 & 4.1 \\
\hline $15-24$ & -3.7 & 26.8 & 40.0 & -54.9 & 16.2 & 5.3 & 41.7 & 6.0 & -3.8 & -12.8 \\
\hline $25-34$ & -19.5 & -0.9 & 41.4 & -60.3 & -21.9 & -11.4 & 15.4 & -4.4 & 1.7 & -11.1 \\
\hline $35-44$ & 9.2 & -4.4 & 17.3 & 40.7 & 2.3 & 14.4 & -8.0 & 14.8 & 4.3 & 13.6 \\
\hline $45-54$ & 10.9 & 4.7 & -31.1 & 27.0 & 8.0 & -2.5 & -0.6 & 12.7 & 5.5 & 9.1 \\
\hline $55-64$ & 13.9 & 13.1 & -32.9 & 11.4 & 14.5 & -3.8 & 24.0 & 20.1 & 7.6 & 9.1 \\
\hline $65-74$ & 3.9 & 9.7 & 5.9 & 7.7 & 10.6 & -2.3 & 47.0 & 13.0 & 8.8 & -0.3 \\
\hline $75-84$ & -4.4 & -4.8 & -5.8 & 9.1 & 2.6 & -3.8 & 24.6 & -2.1 & 5.2 & -3.0 \\
\hline $85+$ & -4.9 & -10.3 & -24.7 & 20.8 & -2.8 & -1.5 & 25.0 & -14.5 & 3.3 & 2.8 \\
\hline
\end{tabular}

aPercent difference $=([\mathrm{DOF}-$ census $] /$ census $) \times 100$. DOF, Department of Finance.

Table 2

DOF-based versus census-based breast cancer incidence rates among non-Hispanic white women (1999-2001)

\begin{tabular}{|c|c|c|c|c|}
\hline County & Average cases per year & $\begin{array}{l}\text { DOF-based incidence rate } \\
(95 \% \mathrm{Cl})^{\mathrm{a}}\end{array}$ & $\begin{array}{l}\text { Census-based incidence rate } \\
(95 \% \mathrm{Cl})^{a}\end{array}$ & Standardized rate ratio \\
\hline Alameda & 575 & $143.3(136.6-150.4)$ & $151.8(144.6-159.2)$ & 0.94 \\
\hline Contra Costa & 592 & $158.0(150.7-165.7)$ & $164.9(157.2-172.9)$ & 0.96 \\
\hline Marin & 245 & $213.6(198.4-229.9)$ & $175.8(163.2-189.5)$ & $1.22^{b}$ \\
\hline San Francisco & 296 & $140.3(131.0-150.7)$ & $159.8(149.2-171.0)$ & 0.88 \\
\hline San Mateo & 406 & $152.3(143.7-161.4)$ & $163.4(154.2-173.2)$ & 0.93 \\
\hline Monterey & 172 & $152.7(139.7-166.9)$ & $150.7(137.7-164.8)$ & 1.01 \\
\hline San Benito & 19 & $114.0(85.9-149.0)$ & $138.7(104.6-182.0)$ & 0.82 \\
\hline Santa Clara & 743 & $143.4(137.5-149.5)$ & $158.6(152.0-165.4)$ & 0.90 \\
\hline Santa Cruz & 161 & $148.2(135.1-162.4)$ & $157.8(143.8-173.0)$ & $0.94^{b}$ \\
\hline Los Angeles & 3587 & $153.8(150.7-156.9)$ & $161.0(157.8-164.3)$ & $0.96^{b}$ \\
\hline
\end{tabular}

alncidence rates are expressed per 100,000 women based on year 2000 population denominators. Data are adjusted to the 2000 US standard. ${ }^{b} p<0.05$. DOF, Department of Finance.

California DOF correlated overall and for selected population strata with counts from the 2000 US census; and to assess how breast cancer incidence rates in selected California counties could be affected by the error of closure between DOF estimates and census counts.

\section{Materials and methods}

\section{Data sources and study population}

At the time of this analysis, 10 counties in California participated in the National Cancer Institute's SEER program (Alameda, Contra Costa, Marin, San Francisco, San Mateo, Monterey, San Benito, Santa Clara, Santa Cruz, and Los Angeles). Data on incident invasive breast cancers diagnosed between 1999 and 2001 in these counties were accessed with public-use SEER data files (based on the SEER 11 Sub for Expanded Races November 2003 submission released April 2004) [1].

Age, sex, and race/ethnicity-specific population data for the year 2000 for counties under analysis were obtained from the DOF and the US Census Bureau [9,11]. Year 2000 DOF estimates used in these analyses were projected based on the 1990 census and were not adjusted to the 2000 census, although DOF data adjusted to the 2000 census are now available. Year 2000 data from the US Census represent actual year 2000 counts.

Analyses were limited to $\mathrm{nHW}$ women. We limited the population to this group to avoid confounding by race/ethnicity; because breast cancer incidence rates are higher among 
$\mathrm{nHW}$ women than among women of any other race, to include breast cancer incidence estimates for Los Angeles County (where only $31 \%$ of the population is $\mathrm{nHW}$ ) and estimates for Marin County (where $79 \%$ of the population is $\mathrm{nHW}$ ) in the same analysis, without accounting for race, could be misleading [5]. There are, however, important differences in the way the DOF and the census categorize race/ethnicity. The 2000 US Census allowed individuals to report up to six distinct ethnicities concurrently to categorize themselves, whereas the DOF stratified the population into five mutually exclusive race categories (white, Hispanic, African-American, Asian Pacific Islander, and American Indian) [10,11]. To control for these differences in race categorization, a US census dataset with bridged race categories was used [12].

\section{Comparison of population data}

Year 2000 population data from the DOF and the census were compared overall and stratified by county, gender, and age group. We examined these stratified groups in order to identify and describe those most likely to be impacted by discrepancies in population estimates.

For all comparisons, 2000 census data were chosen as the standard. Percent differences between data sources can thus be interpreted as the percent by which DOF estimates overestimate or underestimate corresponding census counts. Very small percent differences are to be expected due to the fact that census estimates are based on the population as of 1 April 2000, whereas DOF estimates are based on the population as of the middle of the year $[10,11]$.

\section{Analysis of incidence rates}

For the comparison of breast cancer incidence rates, we included cases of invasive breast cancer (classified as 50.050.9 by the International Classification of Diseases; Oncology, $2^{\text {nd }}$ edition) diagnosed between the years 1999 and 2001 [13]. County-specific incidence rates were age-adjusted using direct-standardization methods, adjusting to the 2000 US standard population [14]. Incidence rates based on year 2000 DOF population estimates, and their corresponding 95\% confidence intervals, were compared to incidence rates based on year 2000 census counts for each county under analysis.

Standardized rate ratios were calculated to compare DOFbased and census-based incidence rates for all counties under analysis. Census-based rates were used as the reference in all regression models, such that rate ratios derived from each of the 10 county-specific models describe the influence of discrepancies between rate denominators independent of the rate numerator.

\section{Results}

Overall, the DOF estimated the size of the year 2000 California population to be $2.3 \%$ larger than was counted by the census. When restricted to $\mathrm{nHW}$ women in the 10 counties under
Figure 2

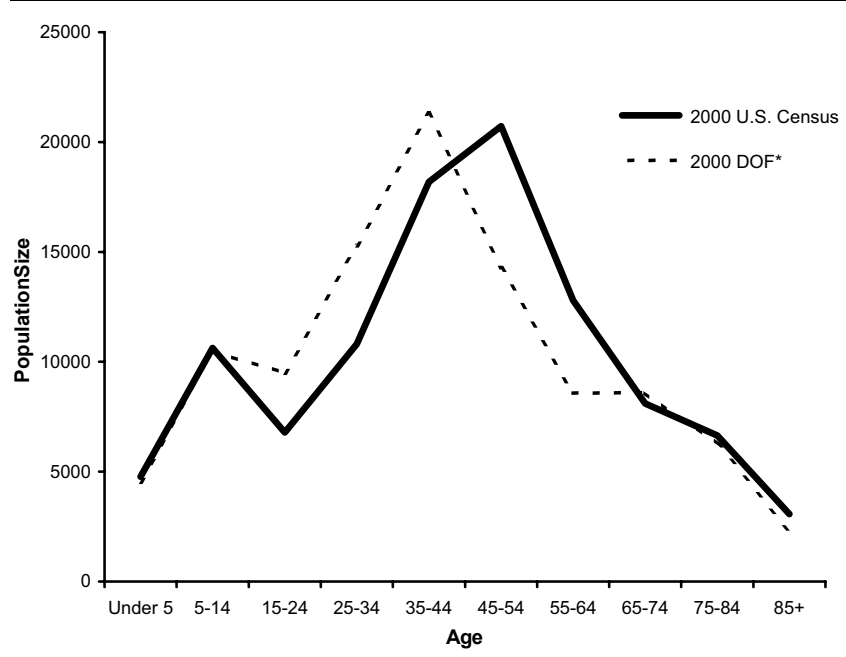

Marin County, California, population estimates for non-Hispanic white women by age and population data source (2000). DOF, Department of Finance.

analysis, DOF population estimates exceeded census counts by approximately $2.9 \%$, ranging from $5.7 \%$ below census counts in San Francisco County to $10.4 \%$ above census counts in San Benito County. Table 1 summarizes discrepancies between population data sources by age strata and county.

When further stratified by age group, DOF and census county population data for $\mathrm{nHW}$ women differed more significantly, although patterns of overestimation and underestimation across age strata differed by county. Percent differences between the two population data sources ranged from $<0.1 \%$ to $64.1 \%$. Discrepancies by age group were largest in San Francisco County, where the percent difference between DOF and census data was more than $30 \%$ in 4 of 10 age groups for $\mathrm{nHW}$ women (ranging from $-60.3 \%$ to $64.1 \%$ ), and in Marin County, where percent differences also exceeded $30 \%$ in 4 of 10 age groups (ranging from $-32.9 \%$ to $41.4 \%$ ). More importantly, however, were discrepancies between population estimates for age groups with the highest incidence of breast cancer; among $\mathrm{nHW}$ women aged 45 years and older, the most substantial population data discrepancies were in Marin County, where DOF population projections for $\mathrm{nHW}$ women aged 45 to 64 years fell below census estimates by approximately $31.7 \%$ and in San Benito County, where DOF estimates for the 55 years and older population exceeded census estimates by $30.6 \%$. Age-specific discrepancies for Marin County are plotted in Fig. 2.

Direct comparison of year 2000 DOF- and census-based ageadjusted incidence rates by county revealed significant differences in estimated incidence rates by population source in three of the ten counties under analysis (Table 2). Breast can- 


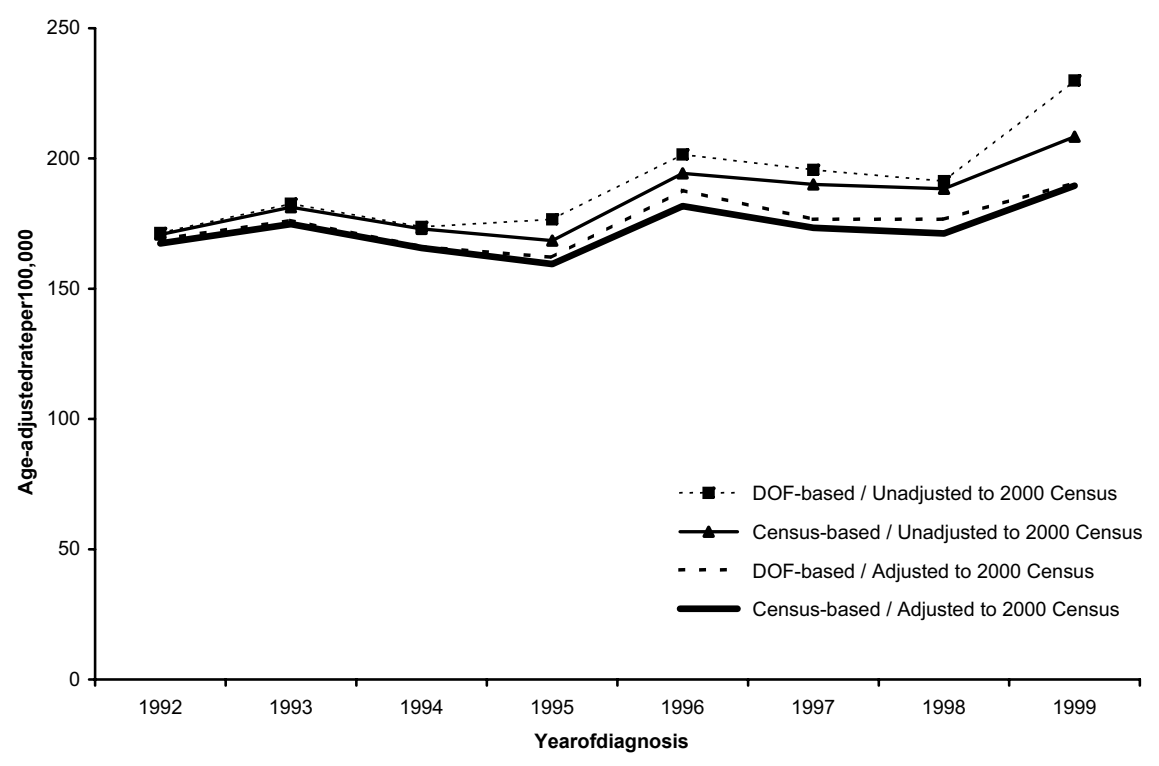

Breast cancer incidence in Marin County, California, by population data source, and adjustment 2000 US census, for $1992-1999$.

cer incidence rates in Santa Clara and Los Angeles counties were significantly lower when based on DOF county population estimates compared to census county population data, adjusting for age: the DOF-based rate was $143.4,95 \% \mathrm{Cl}=$ (137.5-149.5) versus the census-based rate of 158.6, 95\% $\mathrm{Cl}=(152.0-165.4)$ in Santa Clara; and the DOF-based rate was $153.8,95 \% \mathrm{Cl}=(150.7-156.9)$ versus the censusbased rate of $161.0,95 \% \mathrm{Cl}=(157.8-164.3)$ in Los Angeles. Marin County was the only county where the DOF-based rate was significantly higher than the census-based rate: the DOFbased rate was $213.6,95 \% \mathrm{Cl}=(198.4-229.9)$ versus the census-based rate of $175.8,95 \% \mathrm{Cl}=(163.2-189.5))$. The DOF-based rates for Marin County were approximately $22 \%$ higher than census-based rates based on the same numerators.

\section{Discussion}

These analyses have explored the extent to which use of intercensal population projections, extrapolated and 10 years removed from the 1990 census, may have biased breast cancer incidence rates reported in California in the 1990s. DOFbased incidence rates for Marin, Santa Clara, and Los Angeles counties were found to differ significantly from census-based incidence rates: county-specific DOF-based rates were lower than census-based rates in Santa Clara and Los Angeles counties, but higher than census-based rates in Marin County.

Direct comparison of year 2000 DOF and census population data revealed accuracy of DOF projections at the state level, although joint stratification of population estimates by county, gender, race/ethnicity, and age introduced greater discrepancy between population data sources. These discrepancies between stratified population estimates were significant enough to lead to notable differences in breast cancer incidence rates, and can be expected to have a notable effect on other statistics based on DOF intercensal estimates not adjusted to the 2000 census. For example, in the case of San Francisco County, substantial overestimation of the 5-14 year old $\mathrm{nHW}$ female population $(64.1 \%)$ by the DOF compared to the 2000 US census, while having a negligible effect on county breast cancer incidence rates due to the negligible rate of breast cancer among this age group, may be anticipated to have a notable impact on childhood cancer rates.

The fact that differences between population data sources had a significant effect on breast cancer incidence rates in the two largest counties analyzed (Los Angeles, population $9,519,338$, and Santa Clara, population 1,682,585) and the second smallest county analyzed (Marin, population 247,289) suggests that population size is not responsible for variation between census and DOF data. Indeed, no pattern of deviation between the two population data sources is discernable by county size, age distribution, or county urban/rural status. It is possible that methods used by the census and methods employed by the DOF are differentially effective among different populations, or that differing levels of domestic migration explains the discrepancies between these population data sources. The source of these discrepancies, however, remains unknown and was beyond the scope of this analysis.

One limitation to the applicability of this analysis is that DOF intercensal population projections are not used as widely as projections provided by the US census, as DOF projections are only available for counties in the state of California; how- 
ever, problems similar to those noted in this analysis have been noted with the application of census projections [7]. Errors in intercensal population projections provided by the US census for the years 1991 to 1999 were recently implicated as a source of significant overestimation of racial disparities in cancer incidence rates [7,15]. In Marin County, US census intercensal population projections were subject to error of closure problems similar to those identified in DOF projections; census projections, unadjusted to the 2000 US census, substantially underestimated the high risk group of Marin County $\mathrm{nHW}$ women aged 45 to 74 years (data not shown), resulting in an overestimation of the overall incidence rate of breast cancer in the latter years of the 1990s (Fig. 3). Thus, it is likely that similar conclusions would have been reached had census intercensal projections been used rather than DOF intercensal projections. Although methodology used to generate intercensal population projections by both the DOF and the Census Bureau is intricate, complex, and complete, both agencies have produced inaccurate projections. These error of closure problems mean that population data, and incidence rates based on these data, become less reliable as they become further removed from the most recent census.

\section{Conclusion}

The California Cancer Registry, as well as county and local governmental agencies and a broad range of community organizations, must rely on intercensal population projections to estimate health trends, allocate resources, and establish priorities with respect to the populations they serve. Timely surveillance requires that intercensal population projections be used to generate population-based rates and trends as soon as reliable incidence counts become available. This analysis, however, demonstrates that intercensal population projections can differ substantially from later decennial census counts. Although it is unrealistic to recommend that disease surveillance be paused in intercensal years, these data remind us that population denominator quality can have a major impact on the interpretation of health statistics. Health agencies must judge whether aberrant health trends should be acted upon prior to the release of population information that could inform the accuracy of population projections, a process that could take five years or more. The gravity of this problem is magnified in the case of diseases like breast cancer that are the focus of public concern and activism, which intensifies demand for information and public health action.

The results of this analysis support the need for a restructuring of population estimation procedures; perhaps more frequent collection of population counts, particularly in regions experiencing high levels of migration. A 10-year period between population censuses is problematic for accurate projection of the age/gender/race-specific yearly population counts needed for health tracking. Alternatively, government agencies producing population projections would benefit from improvements in ways to make more accurate assumptions regarding the growth and distribution of the population. At the least, more health agencies should develop better ways to describe and quantify the uncertainties in population projections and related bias to consumers of health statistics.

\section{Competing interests}

The author(s) declare that they have no competing interests.

\section{Authors' contributions}

AIP completed all analyses and led the writing. R.R. Ereman was the supervising researcher on this project, helped conceive of the study idea and supervised the writing process. C.A. Clarke assisted with the conception of the study analysis and advised the writing. All authors helped review drafts of the manuscript and interpret study findings.

\section{Acknowledgements}

The authors would like to thank Lee Ann Prebil for her comments and helpful assistance, Dr. Steve Selvin for his assistance, and Jennifer Welle and Michael Musante for their assistance in the analysis.

\section{References}

1. Surveillance, Epidemiology, and End Results (SEER) Program Public-Use Data [http://seer.cancer.gov/publicdata/]

2. Northern California Cancer Center: Data summary of Marin County breast cancer incidence rates [http://www.nccc.org/ news/marindata 011702.html].

3. US Census Bureau: State and County Quickfacts [http://quick facts.census.gov/qfd/].

4. Marin County Department of Health and Human Services: Marin Community Health Survey 2001.

5. Kelsey JL, Bernstein L: Epidemiology and prevention of breast cancer. Annu Rev Public Health 1996, 17:47-67.

6. Clarke CA, Glaser SL, West DW, Ereman RR, Erdmann CA, Barlow JM, Wrensch MR: Breast cancer incidence and mortality trends in an affluent population: Marin County, California, USA 1990-1999. Breast Cancer Res 2002, 4:R13.

7. Boscoe FP, Miller BA: Population estimation error and its impact on 1991-1999 cancer rates. Prof Geographer 2004, 56:516-529.

8. Rosenwaike I, Yaffe N, Sagi PC: The recent decline in mortality of the extreme aged: An analysis of statistical data. Am J Public Health 1980, 70:1074-1080.

9. State of California, Department of Finance: Projected Total Population of California Counties 1990 to 2040 Report 93 P-3 Sacramento, California; 1993.

10. State of California, Department of Finance: County Population Projections with Age, Sex and Race/Ethnic Detail Sacramento, California; 1998.

11. US Census Bureau: Racial and Ethnic Classifications Used in Census 2000 and Beyond [http://www.census.gov/population/ www/socdemo/race/racefactcb.html].

12. National Center for Health Statistics: Bridged-race population estimates for April 1, by county, single-year of age, bridged-race, Hispanic origin, and sex 2000 [http://www.cdc.gov/nchs/about/ major/dvs/popbridge/datadoc.htm\#april2000].

13. Young JL Jr, Roffers SD, Ries LAG, Fritz AG, Hurlbut AA, (eds): SEER Summary Staging Manual - 2000 Codes and Coding Instructions. National Cancer Institute, NIH Pub. No. 01-4969, Bethesda, MD 2001 [http://seer.cancer.gov/tools/ssm].

14. Breslow NE, Day NE: Statistical Methods in Cancer Research The Design and Analysis of Cohort Studies Volume 2. New York: Oxford; 1996.

15. Faulty estimates led $\mathrm{NCl}$ to overstate Black-White cancer disparity in Atlanta. The Cancer Letter 2002, 28:1-5. 